\title{
A Rare Case of Chylothorax Attributed to Metastatic Prostate Carcinoma
}

\section{Hanine Inaty ${ }^{1 *}$ and Maher Tabba ${ }^{2}$}

${ }^{1}$ Department of Internal Medicine at Tufts Medical Center, Boston, Massachusetts, USA

${ }^{2}$ Department of Pulmonary Critical care and sleep medicine at Tufts Medical Center, Boston, Massachusetts, USA

\begin{abstract}
Chylothorax is caused by accumulation of lymphatic fluid into the pleural space. Malignancy and surgical trauma of the thoracic duct are the most common etiologies. Prostate cancer has been rarely reported as a cause of chylothorax. It results from either invasion or compression of the thoracic duct by the metastatic tumor. The mainstay of management is chemoradiation therapy. In this case, we are reporting a patient with metastatic prostate cancer who developed chylothorax while on Dasatinib as part of his chemotherapy regimen. It was not clear if the chylothorax was developed secondary to the metastatic prostate carcinoma or to the chemotherapy.
\end{abstract}

Keywords: Chylothorax; Pleural effusion; Prostate cancer; Dasatinib

\section{Case Report}

A 69 year old male with castration resistant stage IV prostate cancer was started on new chemotherapy regimen including Docetaxel, Prednisone and Dasatinib, presented with progressive shortness of breath. Radiographic images including CT scan of the chest revealed left more than right bilateral pleural effusions (Figure 1). The patient underwent therapeutic left thoracentesis which revealed lymphocytic predominance exudative pleural effusion with elevated triglyceride level $(271 \mathrm{mg} / \mathrm{ml})$. The diagnosis of chylothorax was made. The pleural fluid cytology was positive for metastatic adenocarcinoma cells suggestive of metastatic prostate cancer. Dasatinib therapy was considered a possible alternative etiology of his pleural effusion. Despite the fact dasatinib therapy was started just prior to his current presentation, the chemotherapy was continued without dose adjustment. Given positive cytology for malignant cells, the primary diagnosis was chylothorax due to metastatic prostate carcinoma. No further invasive studies were done at that time. The patient was closely followed in the clinic. His symptoms significantly improved following drainage, and repeat imaging studies showed improvement in tumor burden and no significant reaccumulation of pleural fluid.

\section{Discussion}

Chyle is normally absorbed from the small intestines and drains into the venous circulation via the thoracic duct. Chylothorax is defined as an accumulation of lymphatic fluid into the pleural space. It is characterized by the presence of elevated level of triglyceride above $110 \mathrm{mg} / \mathrm{ml}$ [1]. Malignancies, mostly lymphomas, are the most

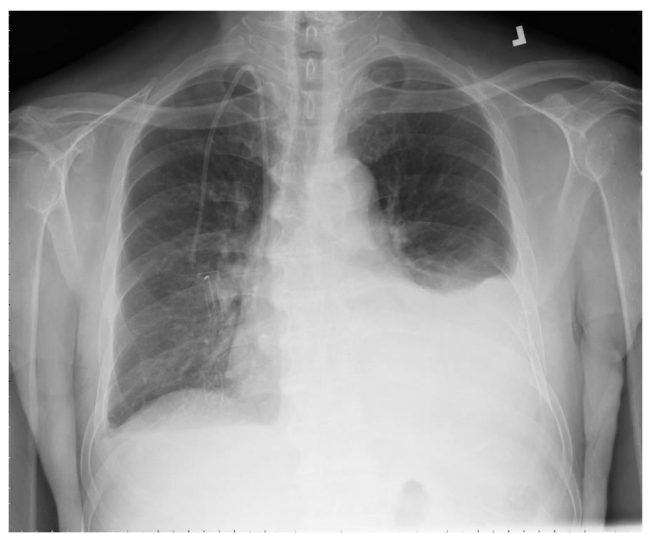

Figure 1: Chest radiogram showing large left sided and small right sided pleural effusions. common causes of chylothorax [1,2]. The mechanism is mainly by lymphangitic spread of the tumor or direct invasion of the thoracic duct $[1,2]$. Metastatic prostate cancer is a very rare cause of chylothorax, only three cases have been reported in the English literature [3-5]. Treatment includes dietary restriction to minimize the chyle production and the lymphatic flow through the thoracic duct such as medium chain fatty acid, octreotide therapy or total parenteral nutrition [1,2]. Chylothorax drainage by frequent thoracentesis such as chest tube or tunneled catheter will put the patient at risk for significant protein loss, electrolytes impairment and lymphopenia and is highly discouraged [4]. Draining more than 1.5 liters per day of chyle, or more than a liter per day for 5 days or draining for more than 2 weeks indicates that conservative measures failed and more invasive therapy should be pursued [1,2]. Surgical ligation of the thoracic duct may be indicated in traumatic causes of chylothorax but is often not successful in cases of malignancies due to difficulty in identifying the site of the leakage given the degree of inflammation [4]. Chemical pleurodesis is usually not successful in chylothorax effusion. Chemoradiation therapy to control the underlying malignancy remains the first line treatment option. Radiation therapy not only controls the disease, but also creates an inflammatory process that leads to fibrosis and obliteration of the leakage site $[4,6,7]$.

Our patient was recently started on Dasatinib therapy for castration resistant prostate cancer shortly prior to his current presentation. Dasatinib is a new tyrosine kinase inhibitor, recently approved for treatment of BCR-ABL positive chronic myelogenous leukemia $[8,9]$. Dasatinib induced chylothorax has been described in leukemia patients especially at high dose. The mechanism is thought to be due to inhibition of platelet derived growth factor receptor expressed in pericytes which has a role in regulation of angiogenesis. Inhibition of this receptor leads to impairment of the vasculature permeability and thus pleural effusions [9]. The treatment would be either reduction of the dose or discontinuation of the drug [8].

${ }^{*}$ Corresponding author: Hanine Inaty, Department of Internal Medicine at Tufts Medical Center, 800 Washington Street, Boston MA 02111, USA, Tel: 857-2102484; E-mail: hinaty@tuftsmedicalcenter.org

Received February 12, 2013; Accepted March 22, 2013; Published March 26 2013

Citation: Inaty H, Tabba M (2013) A Rare Case of Chylothorax Attributed to Metastatic Prostate Carcinoma. J Pulmon Resp Med S14: 002. doi:10.4172/2161105X.S14-002

Copyright: (ㅇ 2013 Inaty $\mathrm{H}$, et al. This is an open-access article distributed under the terms of the Creative Commons Attribution License, which permits unrestricted use, distribution, and reproduction in any medium, provided the original author and source are credited. 
Because of the positive cytology in the pleural fluid analysis, the patient completed the full course of chemotherapy without any change in the dose. The patient had significant clinical response and the pleural effusion resolved. This improvement supports the fact that his pleural effusion was most likely due to the metastatic prostate cancer rather than a side effect of Dasatinib.

In summary, Chylothorax secondary to prostate cancer is very rare. The mainstay of therapy is controlling the underlying malignancy with aggressive chemoradiation therapy.

\section{References}

1. McGrath EE, Blades Z, Anderson PB (2010) Chylothorax: aetiology, diagnosis and therapeutic options. Respir Med 104: 1-8.

2. Nair SK, Petko M, Hayward MP (2007) Aetiology and management of chylothorax in adults. Eur J Cardiothorac Surg 32: 362-369.

3. Cigarral C, Montero A, Salas C, Rodríguez G, de la Torre A (2009) Chylothorax due to metastatic prostate carcinoma: an unusual complication. Clin Trans Oncol 11: 767-769.

4. Little NA, Walther PJ (1985) Chylothorax: a rare complication of metastatic prostatic carcinoma. J Urol 134: 1215-1217.

5. Quinonez A, Halabe J, Avelar F, Lifshitz A, Moreno J, et al. (1989) Chylothorax due to metastatic prostatic carcinoma. Br J Urol 63: 325-327.

6. Heaton RW, Arnold IR, Howard N, Guz A (1987) Successful treatment of chylothorax and superior vena cava obstruction by radiotherapy. Thorax 42 : 153-154.

7. Johnson DW, Klazynski PT, Gordon WH, Russell DA (1986) Mediastina lymphangioma and chylothorax: the role of radiotherapy. Ann Thorac Surg 41 325-328.

8. Masiello D, Gorospe G 3rd, Yang AS (2009) The occurrence and management of fluid retention associated with TKI therapy in CML, with a focus on dasatinib. J Hematol Oncol 2: 46.

9. Kelly K, Swords R, Mahalingam D, Padmanabhan S, Giles FJ (2009) Serosal inflammation (pleural and pericardial effusions) related to tyrosine kinase inhibitors. Target Oncol 4: 99-105. 